\title{
Maximum Hygrostress Response Charts for Drying or Hydrating Elastic, Cylindrical Food with Infinite Biot Number
}

\author{
Kan-ichi HAYAKAWA and Jing HE* \\ Food Science Department, New Jersey Agricultural Experiment Station, Cook College, Rutgers University, CS Dudley Rd-New \\ Brunswick, NJ 08901-8520, USA
}

Received September 1, 1997; Accepted February 2, 1998

\begin{abstract}
Charts were prepared for estimating local maxima or minima of dimensionless, principal stresses of axial, radial, and tangential orientations formed in an elastic, cylindrical food during a drying or hydrating process. For this, analytical solutions derived previously were used. These charts showed that tangential stresses were the most critical for stress cracking during drying processes and radial stresses for hydrating processes. Sample applications of the charts were presented for adjusting process conditions to prevent stress cracking.
\end{abstract}

Keywords: maximum hygrostress, elastic cylinder, moisture diffusion

Moisture distributions are not uniform in food undergoing a drying or hydrating process. This results in nonuiform volumetric shrinkage or expansion which leads to hygrostress formation in most foods. When the hygrostress reaches a critical level, stress cracking occurs. A proper process design is required to prevent cracking. A reliable method for predicting stress formation is useful for the process design since a trial-and-error approach for the process design is costly and time-consuming. Therefore, several researchers have developed such methods (Akiyama et al., 1997; Irudayaraj \& Haghighi, 1993a and b; Itaya et al., 1995; Izumi \& Hayakawa, 1995; Litchfield \& Okos, 1988; Tsukada et al., 1991). While these methods predict accurately the rheological response of food undergoing drying, a simplified, less computation intensive method is useful for preliminary process evaluation although it is based on simplified assumptions on physical and operational conditions.

There are several different criteria for stress crack formation (Jaeger, 1969). Among them, the criterion of critical tensile stress (crack forms when a tensile stress exceeds a critical level) has been validated experimentally (Akiyama et al., 1997; Itaya et al., 1995; Izumi \& Hayakawa, 1995; Tsukada et al., 1991). In view of this criterion, the magnitudes of the maximum tensile stresses formed during a process may be used to examine a potential for crack formation during the process. Therefore, charts for estimating these magnitudes were developed for the elastic food of a plate or spherical shape (Hayakawa \& He, 1997) using published analytical solutions for transient state hygrostress formation (Hayakawa, 1994). The present work is to develop similar charts for cylindrical, elastic food since many foods are cylindrical.

* Current affiliation: Structural and Computational Biology and Molecula Biophysics Program, Baylor College of Medicine, Houston, TX 77030

\section{Materials and Methods}

There is heat and moisture transfer in food undergoing drying processes. However, an isothermal condition establishes shortly after the start of drying since thermal diffusivities of most foods are at least 100 times of moisture diffusivities (D). ${ }^{1)}$ Additionally, the Biot numbers $(B i=h a /$ $D$ ) for most drying processes are much greater than 100 since $D$ values are much less than the values of convective surface mass transfer coefficient (h). It has been known that moisture diffusion with $B i>30$ is virtually identical to that with an infinitely large $B i$.

Although the value of $D$ is highly dependent on food moisture concentration, it may be approximated as constant within a narrow concentration range as many researchers have assumed to determine $D$ values by using analytical diffusion solutions based on constant physical property values.

In view of the above, the present work used analytical solutions, which was derived previously to estimate principal stress formation assuming isothermal diffusion, an infinitely large $B i$ and constant physical property values (Hayakawa, 1994).

Stresses acting on any one location may be resolved into those acting on three mutually orthogonal directions. When stresses are resolved into the principal directions, tensile or compressional stresses in these directions become maximum and no shear stress exists in any of these directions. The analytical solutions used for the present work estimate the principal stresses formed within a cylindrical body.

The analytical solutions use this widely practical sign convention: positive for tension and negative for compression. In view of the critical tensile stress criterion, charts were prepared to estimate positively maximum dimensional stress magnitudes as a function of radial location and processing

\footnotetext{
${ }^{1)}$ All symbols used are defined in the nomenclatures.
} 
time.

The following dimensionless groups were used to simplify the chart development.

$$
\begin{gathered}
\Gamma_{\mathrm{jq}}=2 \sigma_{\mathrm{jt}}(1-\nu) /\left[\beta E\left(W_{\mathrm{o}}-W_{\mathrm{e}}\right)\right] \\
F o_{\mathrm{jq}}=D t_{\mathrm{jt}} / a^{2}
\end{gathered}
$$

where $t=\mathrm{m}$ when $\mathrm{q}=\mathrm{m} \& W_{\mathrm{o}}>W_{\mathrm{e}}$ or when $\mathrm{q}=\mathrm{i} \& W_{\mathrm{o}}<W_{\mathrm{e}}$ $t=\mathrm{i}$ when $\mathrm{q}=\mathrm{m} \& W_{\mathrm{o}}<W_{\mathrm{e}}$ or when $\mathrm{q}=\mathrm{i} \& W_{\mathrm{o}}>W_{\mathrm{e}}$

$$
\rho=r / a
$$

and where subscripts $\mathrm{i}$ and $\mathrm{m}$ signify minimum and maximum respectively.

The locally maximum dimensional tensile stress, $\sigma_{\mathrm{jm}}$, formed during drying (i.e. $W_{\mathrm{o}}>W_{\mathrm{e}}$ ) may be estimated using positively maximum dimensionless stress $\Gamma_{\mathrm{jm}}$ and $\sigma_{\mathrm{jm}}$ formed during hydrating (i.e. $W_{\mathrm{o}}<W_{\mathrm{e}}$ ) using the negatively minimum dimensionless stress $\Gamma_{\mathrm{ji}}$. Therefore, the location dependent $\Gamma_{\mathrm{jm}}$ or $\Gamma_{\mathrm{ji}}$ value of the following orientations were computed together with the processing time for the formation using the published analytical formulae (Hayakawa, 1994): radial, tangential, and axial orientations. The algorithms for the above computation were similar to those described previously (Hayakawa \& He, 1997). The major difference between the present and previous algorithms is the estimation of Bessel functions included in the analytical formulae. This estimation is described briefly in the appendix.

Poisson ratio $(\nu)$ is included in the definition of the dimensionless principal stress, Eq. (1). Additionally, it is included in the formula for estimating $\Gamma_{\zeta}$ (Hayakawa, 1994). Therefore, the following $\nu$ values were assumed for the $\Gamma_{\zeta}$ computations: $0.2,0.3,0.4$ and 0.5 (published $\nu$ 's of foods within the range of 0.2 and 0.5 ; Wainwright et al., 1982).

\section{Results and Discussion}

A numerical analysis revealed that local minima of radially oriented, dimensionless principal stresses $\left(\Gamma_{\rho_{1}}\right)$ are all negative and that there was no local maximum in a $\Gamma_{\rho}$ history at any location and that $\Gamma_{\rho}$ on the surface $(\rho=1)$ remained nil throughout a process.

According to Eq. (1), the sign of dimensional stress $\sigma_{\mathrm{jt}}$ becomes opposite of dimensionless stress $\Gamma_{\mathrm{jq}}$ when $W_{\mathrm{o}}<W_{\mathrm{e}}$ (a hydrating process). Therefore, the figure provides locally maximum tensile dimensional stresses $\left(\sigma_{\mathrm{rm}}\right)$ formed during a hydrating process, noting that a positive principal stress (in this case $\sigma_{\mathrm{r}}$ ) is tensile and a negative compressional. An estimated tensile stress $\left(\sigma_{\mathrm{rm}}\right)$ may be compared against a critical tensile stress for failure $\left(\sigma_{\mathrm{c}}\right)$ to examine the a potential for stress cracking. Since the globally minimum stress $\left(\Gamma_{\rho}{ }^{\mathrm{i}}\right)$ forms along the cylindrical axis $(\rho=0)$, the globally maximum dimensional tensile stress $\rho_{\mathrm{r}}{ }^{\mathrm{m}}$ forms at the same location during a hydrating process(superscripts $\mathrm{i}$ and $\mathrm{m}$ signify globally minimum and maximum, respectively). Noting that $\Gamma_{\rho}{ }^{\mathrm{i}}=-0.47$ from Fig. $1, \sigma_{\mathrm{r}}{ }^{\mathrm{m}}$ may be estimated by:

$$
\sigma_{\mathrm{r}}{ }^{\mathrm{m}}=0.47 \beta E\left(W_{\mathrm{e}}-W_{\mathrm{o}}\right) /[2(1-\nu)]
$$

Figure 2 shows dimensionless time $F o_{\rho \mathrm{m}}$ for $\Gamma \rho_{\mathrm{i}}$ formation. It is zero on the surface (no minimum formed on the surface since $\Gamma_{\rho}$ is initially nil and remains nil) and increases with reduction in $\rho$, the largest $F o_{\rho \mathrm{m}}(0.077)$ on the cylindrical axis.

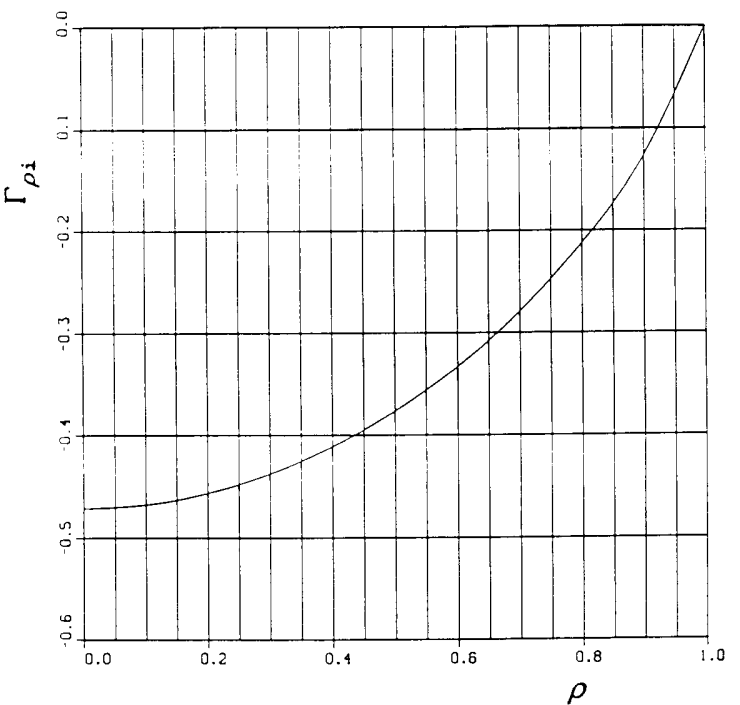

Fig. 1. Location dependent local minimum $\left(\Gamma_{\rho \mathrm{i}}\right)$ of dimensionless radial principal stress.

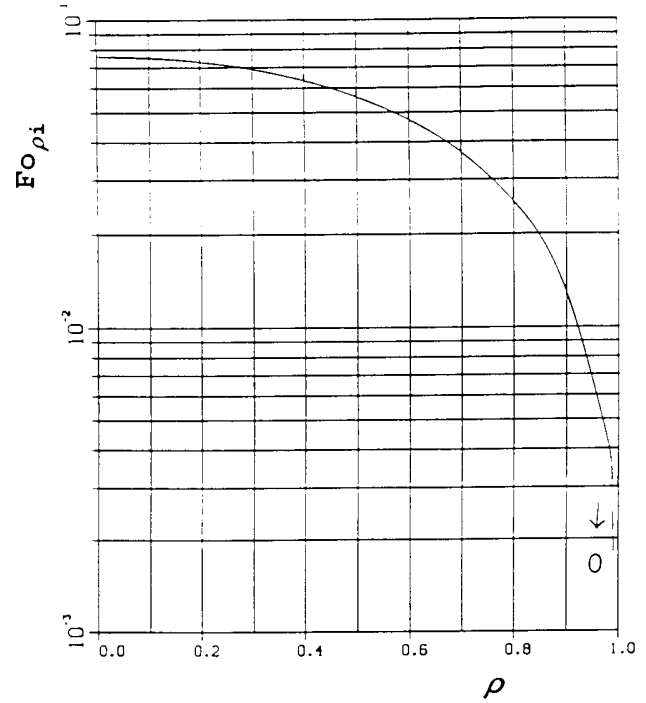

Fig. 2. Location dependent Fourier number $\left(F o_{\rho 1}\right)$ for formation of local minimum of dimensionless radial principal stress.

Since the globally maximum $\sigma_{\mathrm{r}}\left(\sigma_{\mathrm{r}}{ }^{\mathrm{m}}\right)$ forms on the central axis, the dimensional time $t_{\mathrm{r}}^{\mathrm{m}}$ for this formation is estimated by:

$$
t_{\mathrm{r}}^{\mathrm{m}}=0.077 a^{2} / D
$$

Figures 3 and 4 are for the local maxima (Curves A) or minima (Curves B) of dimensionless, principal stress of tangential orientation (the direction of cylindrical angle variable $\phi)$. There are both maximum $\left(\Gamma_{\phi \mathrm{m}}\right)$ and minimum $\left(\Gamma_{\phi \mathrm{i}}\right)$ in a principal stress history at any location within 0.55 $\leq \rho<1$ and the minimum only within $0 \leq \rho<0.55$, Fig. 3 . Figure 4 shows that the local maximum occurs before the local minimum at any location within $0.55 \leq \rho<1$ and that the local minimum forms at any $\rho<0.55$ after the formation of local maximum at any $\rho<0.925$. 


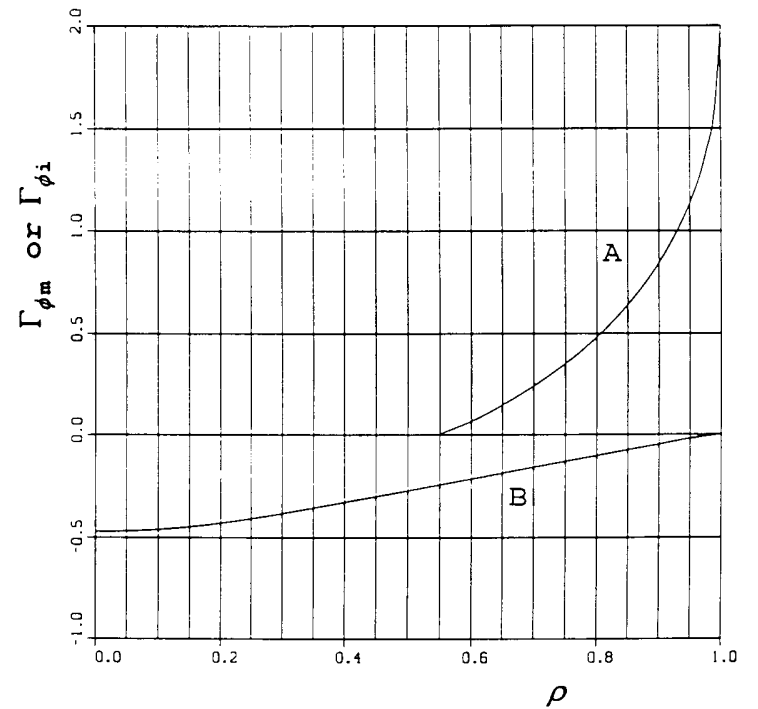

Fig. 3. Location dependent local Maximum $\left(\Gamma_{\phi \mathrm{m}}\right)$ or Minimum $\left(\Gamma_{\phi \mathrm{i}}\right)$ of dimensionless tangential principal stress. Curve $\mathrm{A}$ for local maximum $\left(\Gamma_{\phi \mathrm{m}}\right)$ and Curve $B$ for local minimum $\left(\Gamma_{\phi i}\right)$.

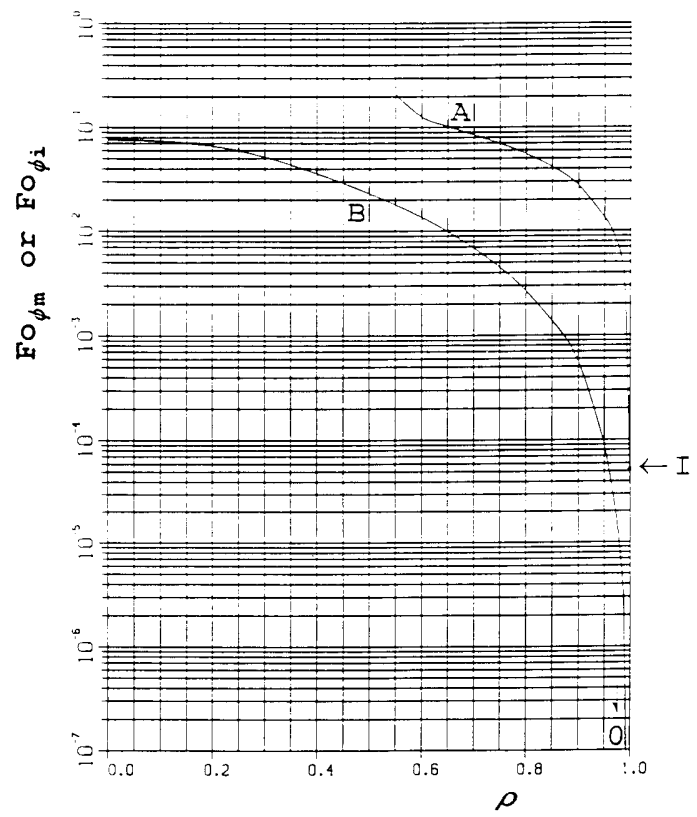

Fig. 4. Location dependent Fourier number $\left(F o_{\phi \mathrm{m}}\right.$ or $\left.F o \phi_{\mathrm{i}}\right)$ for formation of local maximum or minimum of dimensionless tangential principal stress. Curve A for Fourier number for local maximum formation $\left(F o_{\phi \mathrm{m}}\right)$ and Curve B for Fourier number for local minimum formation $\left(F o_{\phi i}\right)$.

Curves $\mathrm{A}$ in Figs. 3 and 4 are useful for drying process evaluation since they estimate locally maximum dimensional stresses (magnitude and time). Curves B are useful for hydrating process evaluation due to a similar reason. Both figures show that the global maximum $\left(\Gamma_{\phi}^{\mathrm{m}}\right)$ is 2.0 which forms on the cylindrical surface at Fourier number $\left(\mathrm{Fo}_{\phi}{ }^{\mathrm{m}}\right)$ of $5.1 \times 10^{-5}$ (point $I$ in Fig. 4) and that the global minimum $\left(\Gamma_{\phi}^{1}\right)$ is -0.47 which forms along the central axis at Fourier number $\left(\mathrm{Fo}_{\phi}{ }^{\mathrm{i}}\right)$ of 0.076 .
The existence of both minimum and maximum as shown in Figs. 3 and 4 reflects the following hygrophysical changes during drying. Shortly after start of drying, surface moisture reduces while there is no moisture loss in a zone adjacent to the surface. Therefore, large hygrostrains form in the surface zone. This leads to mechanical competition between the surface and interior zones, resulting in the formation of large tangential tensile stresses in the surface zone (a zero radial stress on the surface due to no force applied from outside to the body). With the progress of drying, the influence of drying moves inward, resulting in reduced strain magnitudes in the surface zone. This causes reduced surface tensile stresses. With a further progress in drying, the rate of moisture loss in the surface zone reduces. This results in reduced hygroshrinkage in the surface zone while there is increased hygroshrinkage in the interior due to an increased rate of moisture loss. This leads to the formation of compressional, tangential stresses in the interior zone due to mechanical competition between the surface and interior zones.

When the drying process continues further, the locations of both the local maximum tensile stress $\left(\sigma_{\phi \mathrm{m}}\right)$ and maximum compressional stress $\left(\sigma_{\phi \mathrm{i}}\right.$, negative $\left.\sigma_{\phi}\right)$ moves further inward with reduced $\sigma_{\phi \mathrm{m}}$ and increased absolute value of $\sigma_{\phi \mathrm{i}}$. The formation of $\sigma_{\phi 1}$ lags behind $\sigma_{\phi \mathrm{m}}$ formation since the former requires a high drying rate in the interior while the latter requires a high drying rate in the surface zone. When a high drying rate zone reaches the central axis, there is large hygroshrinkage around the axis while greatly reduced or nil shrinkage in surrounding zones, resulting in formation of the globally largest compressional stress in the central zone.

Figures 5 and 6 are related to the stresses of the axial orientation. In Fig. 5, a part of the abscissa, $\rho>0.8$, was enlarged to show details in complex changes in the stresses. Results for a $\nu$ value of 0.5 provide information on limiting hygrostress responses since the $\nu$ values of all soid bodies should be less than 0.5 and since a body with the $\nu$ value of 0.5 is an idealized body that undergoes liquid like deformation (Wainwright et al., 1982). An assumed Poisson ratio $(\nu)$ is shown by each curve of the figures. Figure 5 shows that there is a local maximum and no local minimum in any $\Gamma_{\zeta}$ history at any location. There is an inversion in the influence of $\nu$ on $\Gamma_{\zeta \mathrm{m}}$ in an early stage of a moisture transfer process, Figs. 5 and 6. A local maximum forms shortly after the start of drying on the surface. With the progress in drying, the location of the local maximum moves away from the surface toward the central axis. The greater $\nu$ results in the greater $\Gamma_{\zeta \mathrm{m}}$ on the surface. However, this relationship inverts at a $\rho$ between 0.95 and 1.0. At a $\rho$ below 0.95 , the greater $\nu$ results in the smaller $\Gamma_{\zeta \mathrm{m}}$. This is due to the following hyrophysical changes discussed in terms of product drying.

In the early stage of drying, the influence of drying is limited to a surface zone, with no moisture loss in a zone adjacent to the surface zone. In this case, the larger $\nu$ results in the more fluid like deformation, which is confined in the surface zone, while there is no deformation in the adjacent zone. Therefore, the larger $\nu$ produces the stronger mechanical competition between the surface and adjacent zones, resulting in the larger $\Gamma_{5 \mathrm{~m}}$ on the surface. With the progress of drying, the influence of drying reaches the adjacent zone and 


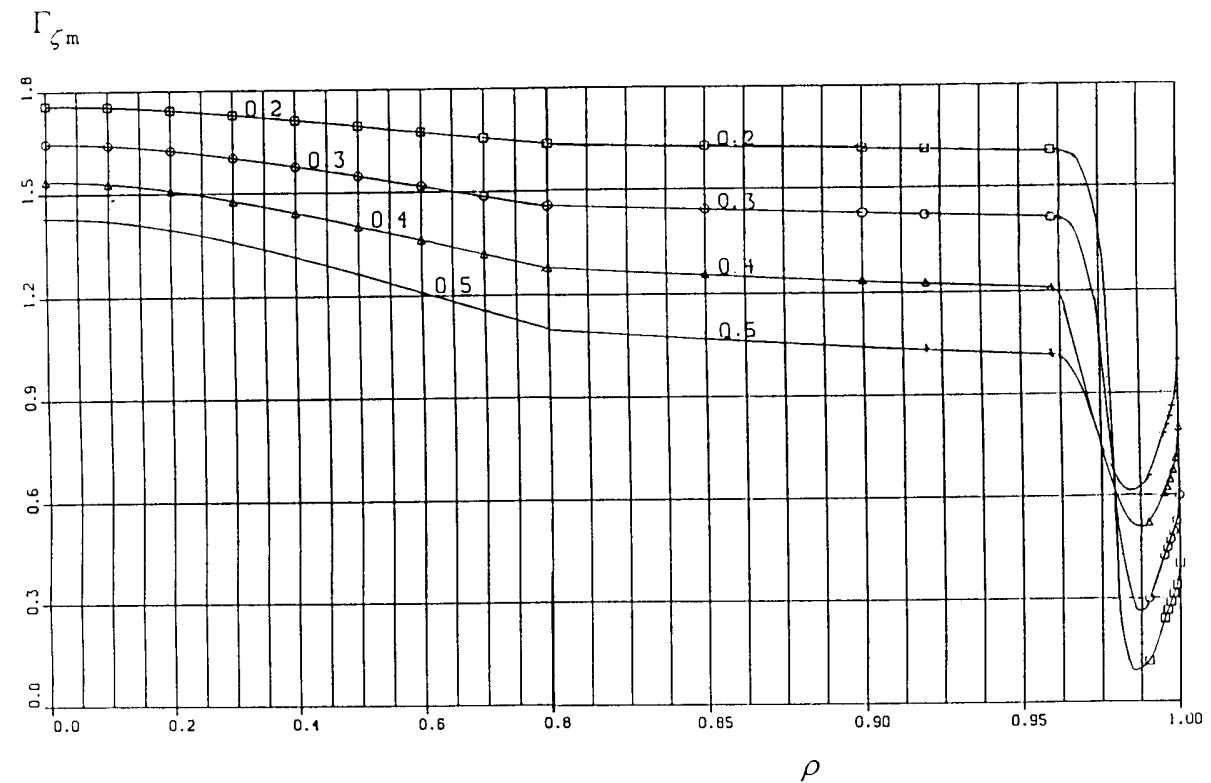

Fig. 5. Location dependent local maximum of dimensionless axial principal stress $\left(\Gamma_{\xi m}\right)$ for different Poisson ratios. Figure by each curve is an applicable Poisson ratio. A part of abscissa, $\rho>0.8$, were enlarged to show details in complex $\Gamma_{\zeta \mathrm{m}}$ changes.

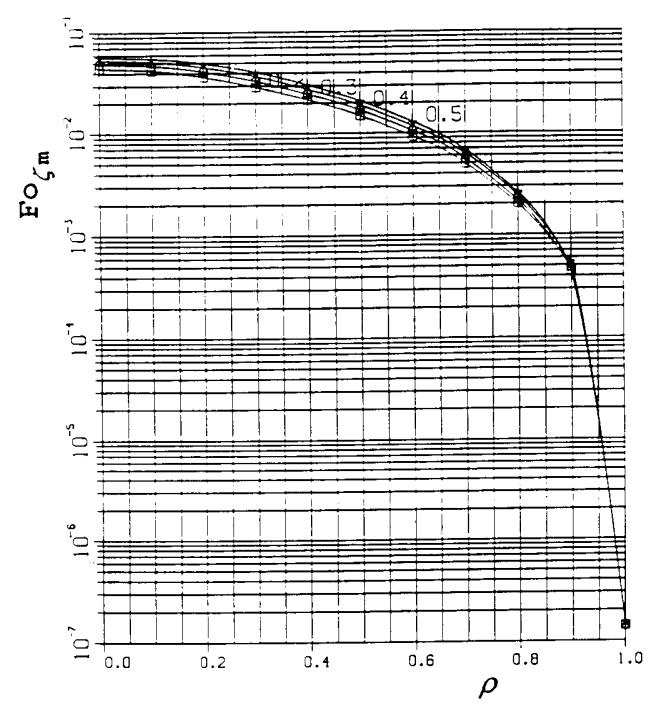

Fig. 6. Location dependent Fourier number $\left(F_{o_{\mathrm{m}}}\right)$ for formation of local maximum of dimensionless axial principal stress. The figure by each curve is an applicable Poisson ratio $(\nu)$, the lowest curve for $\nu$ of 0.2 and the uppermost curve for $\nu$ of 0.5 .

moisture gradient around the surface becomes less distinct, resulting in less mechanical competition between the zones. This leads to the reduced influence of $\nu$ and leads to smaller $\Gamma_{\zeta \mathrm{m}}$. With a further progress in drying, $\Gamma_{\zeta \mathrm{m}}$ increases since there is an increased constraint on axial deformation due to an enlarged zone of drying. The influence of this constraint on $\Gamma_{\zeta \mathrm{m}}$ overcomes the influence of liquidity deformation on $\Gamma_{\zeta \mathrm{m}}$, resulting in the greater $\Gamma_{\zeta \mathrm{m}}$ for the smaller $\nu$.

The global maximum of the dimensionless stress, $\Gamma_{\zeta}{ }^{\mathrm{m}}$, forms along the central axis. The values of $\Gamma_{\zeta} \mathrm{m}$ listed in the ascending order of the assumed $\nu$ 's are: $1.758,1.646,1.536$ and
1.431. According to Fig. 6, these global maxima occur at these Fourier numbers listed in the same order; 0.044, 0.049, 0.053 and 0.058. A cubic polynomial equation was obtained to assist estimating $\Gamma_{\zeta}{ }^{\mathrm{m}}$.

$$
\begin{gathered}
\Gamma_{\mathrm{sm}_{\mathrm{m}}}=6.25 \times 10^{-5}\left(20 \nu^{-7}\right)^{3}+4.375 \times 10^{-4}(20-7)^{2} \\
-0.0550625 \times(20-7)+1.5905625
\end{gathered}
$$

Since the coefficients of Eq. (6) were estimated analytically, the given number of digits should be retained for the estimation. An estimated $\Gamma_{\zeta}{ }^{\mathrm{m}}$ should be truncated to the first four digits.

According to Figs. 3 and 5, the global maximum of dimensionless, tangential principal stress $\left(\Gamma_{\phi}{ }^{\mathrm{m}}\right)$ is greater than the global maximum of axial stress $\left(\Gamma_{\xi}^{\mathrm{m}}\right)$. Therefore, $\Gamma_{\phi}{ }^{m}$ is critical for drying processes. Depending upon the value of a dimensional critical tensile stress for failure $\left(\sigma_{c}\right)$, cracks could form on the surface shortly after start of drying and could propagate inward with the progress of drying. According to experimental observation, the orientation of a stress crack was orthogonal to the orientation of a principal stress responsible for cracking (Akiyama et al, 1997; Itaya et al., 1995; Izumi et al., 1995; Tsukada et al., 1991). Therefore, if a crack forms on the surface, it is oriented axially (orthogonal to tangential direction). With the progress of drying the location of local maximum of tangential stress $\sigma_{\zeta \mathrm{m}}$ or $\Gamma_{\zeta \mathrm{m}}$ moves inward, away from the surface, with a sharply reduced stress magnitude (Figs. 3 and 4). This is accompanied by an increase in local maximum of axial stress $\sigma_{z \mathrm{~m}}$ or $\Gamma_{\mathrm{sm}}$ (Figs. 5 and 6). The value of $\sigma_{z m}$ becomes greater than $\sigma_{\phi \mathrm{m}}$ below a $\rho$ value between 0.87 and 0.96 . A stress crack could form at a location where $\sigma_{\mathrm{zm}}$ exceeds $\sigma_{\mathrm{c}}$. This crack is ring-like, encircling the cylindrical axis (orthogonal to the axial direction). 
Sample applications of the developed charts are given below. Physical properties used for the applications are identical to those used in the previous paper (Hayakawa \& He, 1997). $E=360$ or $1.10 \times 10^{5} \mathrm{kPa}, D=4.0 \times 10^{-11} \mathrm{~m}^{2} / \mathrm{s}, W_{\mathrm{o}}=$ $0.4 \mathrm{~kg}$ water $/ \mathrm{kg}$ dry solid, $W_{\mathrm{e}}=0.08 \mathrm{~kg}$ water $/ \mathrm{kg}$ dry solid, $\beta=0.31, \sigma_{\mathrm{c}}=50 \mathrm{kPa}$ (for $E$ of $360 \mathrm{kPa}$ ) or $1000 \mathrm{kPa}$ (for $E$ of $\left.1.10 \times 10^{5} \mathrm{kPa}\right), \nu=0.3$.

The value of critical dimensionless stress, $\Gamma_{c}$, is estimated using an equation similar to Eq. (1) $\left(\sigma_{\mathrm{jjt}}=\sigma_{\mathrm{c}}=50\right)$ and the above listed property values. With $\sigma_{\mathrm{c}}$ value of $50 \mathrm{kPa}$ and $E$ of $360 \mathrm{kPa}$, one obtains that $\Gamma_{\mathrm{c}}=1.96$. This value is compared to the local maximums given in Fig. 1,3 , and $5 . \Gamma_{\phi \mathrm{m}}$ exceeds 1.96 at $\rho=1$ and in the close vicinity of this $\rho$ according to Fig. 5. Therefore, shortly after the start of drying, cracks form on the surface and they propagate slightly inward with progress of drying. The cracks are oriented axially (orthogonal to the tangential direction). This crack formation may be prevented, reducing $W_{\mathrm{o}}$ slightly to increase $\Gamma_{\mathrm{c}}$ over 2.0 (the global maximum of $\left.\Gamma_{\phi}\right)$ noting that $\Gamma_{\mathrm{c}}=2 \sigma_{\mathrm{c}}(1-\nu) /[\beta E$ $\left.\left(W_{\mathrm{o}}-W_{\mathrm{e}}\right)\right]$. An estimated $W_{\mathrm{o}}$ is $0.394(=0.08+(0.4-0.08) \times$ $1.92 / 2.00)$.

When the values of $\sigma_{\mathrm{c}}$ and $E$ are 1000 and $1.10 \times 10^{5} \mathrm{kPa}$, respectively, one obtains that $\Gamma_{\mathrm{c}}=0.128$. This value is compared to local maximums given in the figures.

The values of $\Gamma_{\phi \mathrm{m}}$ exceeds 0.128 when $0.64 \leq \rho \leq 1$ according to Fig. 3. Therefore, axially oriented stresses form on the surface and likely propagates up to the $\rho$ value of 0.64 . When $\Gamma_{c}$ is compared to $\Gamma_{\zeta \mathrm{m}}$ (Fig. 5), $\Gamma_{\mathrm{c}}<\Gamma_{\zeta \mathrm{m}}$ at all locations. Therefore, ring-shape cracks formed on the surface propagate all way up to the center (complete disintegration). To eliminate cracking, one needs to increase $\Gamma_{\mathrm{c}}$ up to at least 2.0 $\left(\Gamma_{\phi}^{\mathrm{m}}\right)$ adjusting drying conditions and/or modifying food properties. A multifaceted approach is required for this large increase of $\Gamma_{\mathrm{c}}$ from 0.128 to 2.0 . It is assumed that $E$ and $W_{\mathrm{o}}$ are reduced to $90000 \mathrm{kPa}$ and $0.38 \mathrm{~kg} / \mathrm{kg}$, respectively, through product reformulation. With this reduction, a required $W_{\mathrm{e}}$ is estimated using Eq. (1). $W_{\mathrm{e}}=0.129 \mathrm{~kg} / \mathrm{kg}$ $\left(=0.38-2 \times 10^{3} \times 0.7 /(0.31 \times 90000 \times 2)\right)$. Drier air humidity corresponding to this $W_{\mathrm{e}}$ may be estimated from a sorption isotherm of the product. Assume that the above estimated $W_{\mathrm{e}}$ is higher than the final target moisture level of product. One needs to reduce $W_{\mathrm{e}}$ step functionally when product moisture reaches to a level close to 0.129 . Assume that this level is 0.130 . The moisture distribution in the product is virtually uniform at this time of the step functional $W_{\mathrm{e}}$ reduction since $0.130 \simeq$ 0.129 (the estimated $W_{\mathrm{e}}$ ). Therefore, using $W_{\mathrm{o}}$ of 0.130 , the next $W_{\mathrm{e}}$ is estimated through evaluations similar to what is presented above. This turned out to be a negative value $(-0.121)$. Since the minimum $W_{\mathrm{e}}$ is equal to or greater than zero and since minimum $\mathrm{RH}$ is $0 \%$, one may select the lowest humidity level of drier air for the second phase of drying with no problem of stress cracking.

\section{Conclusions}

Charts were developed for estimating location dependent, maximum principal tensile stresses formed in an elastic cylindrical food undergoing an isothermal drying or hydrating process. According to these charts, the global maximum of tangential principal stress formed on the surface is most critical for preventing stress crack formation during drying. For a hydrating process, the global maximum of radial principal stress formed on the central axis is most critical to prevent cracking.

\section{Nomenclatures}

a cylindrical radius $(\mathrm{m})$

$B i=h a / D$. Biot number (-)

$D$ mass diffusivity $\left(\mathrm{m}^{2} / \mathrm{s}\right)$

$E \quad$ Young modulus $(\mathrm{Pa})$

$F o=D t / a^{2}$. Fourier number $(-)$

$h$ convective surface mass transfer coefficient $(\mathrm{m} / \mathrm{s})$

$l$ linear dimension $(\mathrm{m})$

$\Delta l$ change in linear dimension due to moisture concentration change $(\mathrm{m})$

$r$ radial location variable $(\mathrm{m})$

$t$ time variable (s)

$W$ moisture concentration ( $\mathrm{kg} \mathrm{H} \mathrm{H}_{2} \mathrm{O} / \mathrm{kg}$ dry solid)

$\beta$ hygro-shrinkage coefficient defined as shrinkage per unit original linear dimension and per unit moisture concentration change or $\Delta l /\left(l_{0} \Delta W\right)\left(\mathrm{kg}\right.$ dry solid $\left./ \mathrm{kg} \mathrm{H} \mathrm{H}_{2} \mathrm{O}\right)$.

$\Gamma=2 \sigma(1-\nu) /\left[\beta E\left(W_{\mathrm{o}}-W_{\mathrm{e}}\right)\right]$. Dimensionless stress. The first subscript defines stress orientation and second subscript defines the local minimum or maximum. The superscript, if appended, defines the global minimum as maximum $(-)$.

$\sigma$ dimensional stress. The first subscript defines stress orientation and the second subscript defines the local minimum or maximum. The superscript, if appended, defines the global minimum or maximum $(\mathrm{Pa})$.

$\nu \quad$ Poisson ration $(-)$

$\rho=r / a$, dimensionless, radial location variable.

\section{Subscripts}

c critical

e equilibrium

i local minimum

j dummy subscript

m local maximum

o initial

q dummy subscript

$r$ radial (appended to $\sigma$ )

t dummy subscript

$\mathrm{z}$ axial (appended to $\sigma$ )

$\phi$ tangential (oriented to cylindrical angle variable direction, appended to $\sigma$ and $\Gamma$ )

$\xi$ axial (appended to $\Gamma$ )

$\rho \quad$ radial (appended to $\Gamma$ )

\section{Superscript}

i Negatively, global minimum (minimum or maximum absolute value in an entire location and time variable domain).

$\mathrm{m}$ Positively, global maximum (maximum in an entire location and time variable domain).

Acknowledgments This material is based on work supported in part by USDA NRI competitive Grants Program (value added program) Award No. 94-37500-0683, The Center for Advanced Food Technol- 
ogy, a New Jersey Commission on Science and Technology Center, NSF Advanced Computing Resources grant administered at the Pittsburgh Supercomputing Center, New Jersey State Fund, U.S. Hatch Act Fund, and Rutgers University Computing Service Center computer time. New Jersey Agricultural Experiment Station Publication Nos. D10973-1-97 and D10103-1-97.

\section{References}

Abramowtiz, M. and Stegan, I.A. (Eds.) (1964). "Handbook of Mathematical Functions with Formulas, Graphs, and Mathematical Tables," Superintendent of Documents, U.S. Government Printing Office, Washington, D.C, pp. 370 \& 371.

Anonymous. (1991). "FORTRAN Subroutines for Mathematical Applications," IMSL Inc., Houston, TX, pp. 99 \& 101.

Akiyama, T., Liu, H., and Hayakawa, K. (1997). Hygrostress-multicrack formation and propagation in cylindrical viscoelastic food undergoing heat and moisture transfer process. Int. J. Heat Mass Transfer, 40, 1601-1609.

Hayakawa, K. (1994). Analytical solutions for estimating transient state hygrostress formation in elastic food of simple shapes. Lebensm. Wiss. Technol. 27, 232-236.

Hayakawa, K. and He, J. (1997). Maximum hygrostress response charts for drying or hydrating elastic food with infinite Biot number. Spherical or plate food. Lebensm. Wiss. Technol. 30, 773-777

Irudayaraj, J. and Haghighi, K. (1993a). Stress analysis of viscoelastic material during drying-I. Theory and finite element formulation. Drying Technol., 11, 901-927.

Irudayaraj, J., Haghighi, K. and Stroshine, R. (1993b). Stress analysis of viscoelastic material during drying. -Il. Application to grain kernels. Drying Technol., 11, 929-959.

Itaya, Y., Kobayashi, T. and Hayakawa, K. (1995). Three dimensional heat and moisture transfer with viscoelastic strain-stress formation in composite food during drying. Int. J. Heat Mass Transfer, $\mathbf{3 8}$ $1173-1185$.

Izumi, M. and Hayakawa, K. (1995). Heat and moisture transfer and hygrostress crack formation and propagation in cylindrical, elasto- plastic food. Int. J. Heat Mass Transfer, 38, 1033-1041.

Jaeger, J. C. (1969). "Elasticity, Fracture and Flow with Engineering and Geological Applications," Halsted Press, New York, Chapter 20.

Litchfield, J.G. and Okos, M.R. (1988). Prediction of corn kernel stress and breakage induced by drying, tempering and cooking. Trans. $A S A E, 31,585-594$.

Shoup, T.E. (1979). "A Practical Guide to Computer Methods for Engineers," Prentice-Hall, Englewood Cliffs, NJ, pp. 148-150.

Tsukada, T., Sakai, N. and Hayakawa, K. (1991). Computerized model for strain-stress analysis of food undergoing a simultaneous heat and mass transfer process. J. Food. Sci., 56, 1436-1445.

Wainwright, S.A., Briggs, W.D., Cunney, J.D., and Gosline, J.M. (1982). "Mechanical Design in Organisms," Princeton University Press, Princeton, NJ, pp. 11, 12.

\section{Appendix}

Multiple zeros $\left(p_{\mathrm{k}}, k=1,2,3 \ldots\right)$ of the zeroth order J-Bessel function, $J_{0}(x)$, are included in the analytical solutions. Since one needs to estimate 500 or more roots for estimating hygrostresses at a very small dimensionless time, $F_{0}$, and since no published mathematical table showed this many roots, a computer program subroutine was prepared to estimate $p_{\mathrm{k}}$. In this subroutine, values of $J_{0}(x)$ were estimated using a published subroutine (Anonymous, 1991) and approximate roots were estimated using published formula (Abramowtiz \& Stegan, 1964) to initiate iterative computations. The estimated roots were refined iteratively applying a golden search method (Shoup, 1971).

Values of the first order J-Based function at $p_{\mathrm{k}}$ are included additionally in the analytical solution. These values were estimated using a published subroutine (Anonymous, 1991) 